\title{
Post-partum : un moment clé Innovation et rigueur des interventions professionnelles
}

\author{
F. Vendittelli · F. Molénat
}

(C) Lavoisier SAS 2019

Le séjour hospitalier en post-partum a longtemps été difficile à appréhender avec rigueur, pour de multiples raisons. Que ce soit à propos de l'allaitement ou du fameux baby-blues, l'imaginaire, la subjectivité, le flou ont laissé leur empreinte, et beaucoup s'en plaignent, tant du côté des parents que des soignants. L'absence de pathologie - sauf exception génère une absence de cadre précis qui aiderait à définir les rôles et le tempo des actions.

Pourtant, ce séjour devenu très court, sauf indication rare, révèle s'y l'on y prête attention - comme le font actuellement de nombreuses équipes de soins — une intensité sensorielle et émotionnelle, qui souvent peut être masquée : chez la mère, par la sidération d'un accouchement et la fatigue subséquente ; chez les professionnels, par le respect d'une intimité familiale qui brouillerait l'évaluation d'une proximité adéquate à organiser ; et chez le partenaire qui est souvent l'oublié du post-partum, bien sûr, par la durée si courte d'hospitalisation. La cohérence des interventions « soignantes » constitue un enjeu à structurer selon les terrains. Un travail conjoint des sociétés médicales et associations en périnatalité y a été consacré tout récemment. Même si le manque de temps est évoqué de manière cruciale en de

\footnotetext{
F. Vendittelli $(\bowtie)$

Association des utilisateurs de dossiers informatisés en pédiatrie obstétrique et gynécologie (Audipog),

faculté de médecine René-Théophile-Hyacinthe-Laennec, F-69372 Lyon cedex 08, France

e-mail : fvendittelli@chu-clermontferrand.fr
}

CNRS, SIGMA Clermont, institut Pascal, CHU de ClermontFerrand,

université Clermont-Auvergne, F-63000 Clermont-Ferrand, France

Réseau de santé en périnatalité d'Auvergne, CHU de Clermont-Ferrand, F-60003 Clermont-Ferrand cedex 1, France

F. Molénat $(\square)$

Association de formation et de recherche sur l'enfant et son environnement, Montpellier,

11 rue des Hospice, F-34090 Montpellier, France

e-mail : molenatfrancoise@gmail.com nombreux lieux, les effets peuvent en être majorés par la difficulté à répartir les énergies de manière efficace, ce qui met en jeu l'ensemble du parcours périnatal.

Moment clé en effet, car les travaux déjà anciens issus de l'éthologie animale, adaptés à l'humain, ont démontré l'impact à moyen terme des contacts entre la mère et l'enfant. La notion anglo-saxonne de « compétences » du bébé, médiatisée par la série télévisée Le bébé est une personne, avait modifié le regard sur cette étape précoce de la vie. Des bouleversements considérables ont favorisé les premières rencontres, dont on sait que l'empreinte peut marquer durablement le psychisme parental, et donc le style d'interaction qui en découlera. La genèse des troubles précoces d'attachement le démontre dans le quotidien des consultations ultérieures. Que d'épisodes douloureux, de séquelles émotionnelles liées aux différentes étapes du parcours obstétrical, de silences enfouissant bien profond les moments difficiles intriqués aux expériences plus anciennes des parents révèlent la nécessité d'ouvrir le dialogue au plus tôt, dès la grossesse, pour que, dans le continuum relationnel amélioré par la coordination des soins, les mouvements intérieurs des mères et des pères puissent se dire, avec leur charge fréquente de culpabilité et d'angoisse.

La notion d'anticipation prend là tout son sens. De même qu'une naissance sereine ne s'improvise pas, tant pour la mère que pour le père, le séjour en post-partum ne permet guère de détecter et de prévenir les aléas de relations troublées. Encore moins d'organiser un étayage ou simplement un suivi précoce du bébé, s'il s'avère nécessaire, dans une temporalité ajustée au rythme de l'enfant et/ou des parents. Dans les situations de fragilité familiale, la phrase classique « on verra comment ça se passe » devient obsolète. Quand on « voit », il est hélas bien tard pour redresser ce qui aurait pu se faire à peu de frais. Les documents vidéoscopés montrant les troubles acquis chez des enfants plus âgés sont éloquents. Il s'agit donc d'intégrer dans le continuum périnatal ce temps.

L'intérêt est bien là, face à ce qui se noue de manière plus ou moins heureuse dans les émotions de chaque partenaire, dans le jeu des places nouvelles autour du bébé, dans l'adaptation mutuelle des parents avec le nouveau-né. Les 
connaissances récentes sur la plasticité cérébrale à ces stades de la vie, illustrées par l'influence des interactions sur la synaptogenèse, donnent un nouvel élan à la recherche clinique. De même, l'épigénétique portée par le concept des « 1000 premiers jours » stimule les efforts pour mettre à disposition du petit humain ce qui peut influencer la qualité de son organisation future. Le constat se généralise que nous entrons dans une phase nouvelle, où l'on voit se rejoindre divers courants de pensée, issus de champs disciplinaires longtemps éloignés, voire opposés. Cette confluence ouvre de nouveaux espoirs. Une prévention trouverait là ses lettres de noblesse dans le dépassement d'un clivage délétère entre nature et culture, entre corps et esprit, reconnu désormais de manière large.

Place est faite ainsi aux nouvelles expériences sur les terrains. La généralisation des collaborations entre les champs d'exercice fut stimulée par le diagnostic anténatal, par les progrès de la réanimation anténatale, plus lentement par la nécessaire convergence des actions face aux situations de grande vulnérabilité parentale. De ce rapprochement sont nés et s'amplifient des concepts cliniques novateurs. L'approche collective, en offrant un environnement humain porteur via les actions coordonnées, peut changer un destin. Mais elle ne s'improvise pas. C'est pourquoi, dans ce dossier, sont proposées réflexions et tentatives en vue de potentialiser les efforts des soignants, pour que ces quelques jours du post-partum soient l'occasion de ponctuer sans entrave une dynamique de vie. Aboutissement des étapes de gestation et d'accouchement, passage bref et lourd de conséquences, ouverture vers une meilleure prise en compte des besoins dans les semaines à venir : chez le nouveau-né dont les manifestations précoces peuvent alerter, chez les mères dans l'achèvement du processus de gestation, chez le père dans une place à construire.

Une fois posé le cadre médical du séjour par F. Vendittelli seront présentées les études et pratiques innovantes. Intérêt de se rapprocher du vécu maternel au travers d'un entretien postnatal (équipe du CHUV à Genève). Confronter l'état émotionnel de la mère et les représentations des sagesfemmes autour du blues du post-partum, par M. Roux et J. Wendland. Ingrid Bayot, sage-femme, propose son concept de "quatrième trimestre de la grossesse ", période de « dégestation » dans une conviction affirmée que l'attention postnaissance doit s'améliorer. R.M. Toubin, pédopsychiatre, associée au pédiatre A. Jacquot et au gynécologueobstétricien P. Boulot, expose une pratique innovante en post-partum immédiat auprès de parents très inquiets sur l'état du bébé du fait de leurs propres angoisses anténatales. Reine Vanderlinden, psychologue périnatale à Bruxelles, décrit l'évolution en cours sur la place fondamentale que peut occuper le père dans le processus de naissance et son impact pour l'avenir de l'enfant.

Puisse ce numéro spécial autour du post-partum faire engager les professionnels de la périnatalité à penser la prise en charge des femmes, du partenaire et de leurs enfants autrement que par l'approche stéréotypée, tayloriste qui remonte au $\mathrm{xx}^{\mathrm{e}}$ siècle. Un suivi adapté et individualisé aux besoins repérés de la femme et de son enfant, mais aussi de son partenaire, voilà le nouveau challenge à relever par les professionnels du $\mathrm{xxI}^{\mathrm{e}}$ siècle. 